\title{
Mobile Illuminance Measurement of Road Lighting
}

\begin{abstract}
Paper deals with technical solution of mobile measurement of public lighting project. Each part of the measuring device is well described. Various kinds of measured and gathered data are listed as well as the way of their processing and further utilization. The use of special correction function is indicated. Finally the paper deals with visualization and graphic design of measured values and measuring uncertainty estimation.
\end{abstract}

Key words: public lighting, mobile measurement of illuminance, ArcGIS.

\section{Purpose of the device}

The project Mobile Illuminance Measurement of Road Lighting was set up as a response to public lighting providers' request. The objective was to create a method and a measuring device in order to be able to make an easy way verification of the public lighting quality. Although the measuring principle comes out partly from a technical standard [1] (under certain conditions mobile measurement is allowed in another part of this standard [2]) it is not the aim of this device to meet all standard requirements. Thus the results can't be quantitatively compared with the results of the measurement according to the mentioned standard. The meaning of this device is to define the public lighting quality of each street in the whole territorial unit (city, district, township,...) with minimal measurement uncertainty. The result of such measuring is a statement of the street lighting condition (too less light, too much light and well illuminated). In the first two cases a proper measurement according to the standard requirements could be done at the chosen parts of the street afterwards.

\section{Technical description}

Figure 1 shows a schematic description of the device. The measuring device is based on a passenger car with a special roof rack. On this rack four measuring photocells are situated each in one corner. These photocells are in the class $B$ with resolution $0,01 \mathrm{Ix}$ and range from 0,05 to $12500 \mathrm{~lx}$. The distance between photocells is $1 \mathrm{~m}$ crosswise and 1,5 m lengthwise. The photocells are located so they are able to measure the horizontal part of the illuminance in the height of $1,6 \mathrm{~m}$ above the road surface. A temperature and humidity detector is also placed on the rack. There were several reasons why to put the measuring photocells on the roof rack. The main reason was the fact that being placed on the roof rack the photocells aren't shaded by the car itself. The effect of the majority of the other lighting sources (especially the car lights) is also eliminated. These negative influences would lead to measurement uncertainty increase in case they will be placed for instance in the front or behind the car close to the bumper. The disadvantage of the used solution is a need for special correction functions use (see later).

On the roof rack close to each measuring photocell a removable head of calibration photometer is placed. Before the beginning of each measurement every single photocel is calibrated. Calibration photometer is $A$ classed with range from $0,001 \mathrm{~lx}$ to $360 \mathrm{klx}$.

Measuring photocells and the temperature and humidity detector are connected by extension cables with a measuring multimetr placed inside the vehicle. Multimetr (and also a calibration photometer during a calibration) is connected with a notebook placed in front of the passenger's seat. There is a special SW in this notebook used to gather continuously the values of the illuminance and other information set by the crew. GPS module connected to the notebook assigns to every single measuring it's location. A diagnostic unit OBD II is connected into the vehicle's bus placed under a dashboard. Using a Bluetooth connection this OBD unit is providing to the SW information about wheel rotation. Therefore it is possible to make a measurement with constant longitudinal spacing independently on the GPS.

An external screen is also connected to the notebook. A map showing adjacent streets, measured and not measured segment of the roads are displayed on this screen. On a windscreen an in-car camera is mounted. It records the measurement itself, most of all the character of measured street and the public lighting system. This record is automatically stored in the notebook. Behind the back wind screen there is an in-car flashing beacon. All devices inside the car are connected to the car battery.
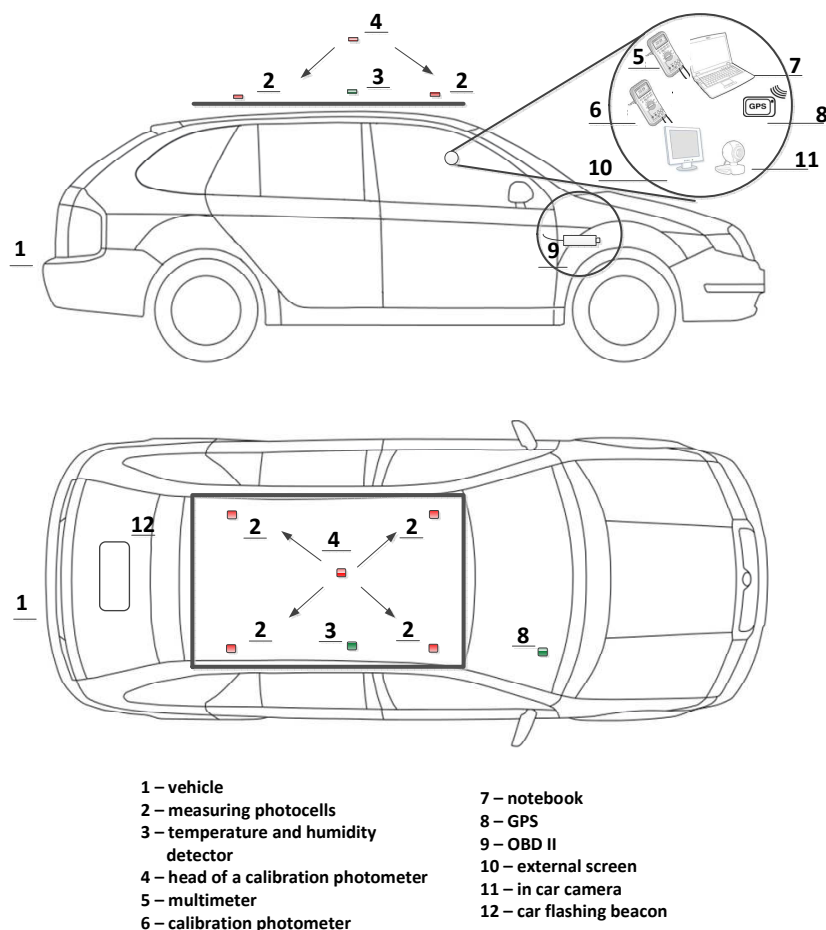

Fig. 1. Technical equipment schema

\section{Measured streets}

Primarily this measuring device is supposed to be applied at the road with automobile traffic, practically it is able to be used everywhere where the measuring car fits.

In spite of the real lighting class, according to the technical report [3] and its requirements, every measured 
road segment is classified as a CE class and the only evaluated quantity is average horizontal illuminance. Qualitative lighting parameters such longitudinal or overall uniformity are not evaluated. Road segments originally classified as other lighting classes are evaluated according to a conversion table from [3].

During the measurement itself a measured street is divided into several logic segments while the number of such segments in one street is greater or equal to one. The end of the street automatically means the end of the logic segment. It is not allowed to place one logic segment between two or more different streets. Logic segment is the part of the street where following parameters are constant:

- Width of the traffic lane

- Height of the lighting point

- Overhang

- Lighting system type

- Ambient brightness

- Traffic intensity

- Conflict area

- Parking vehicles

- Number of crossing

These parameters are used for creating:

- Special correction functions

- Lighting class of measured logic segment

\section{The measurement}

Data gathering is ensured by a two man technically trained crew - the driver and the operator. Before the measurement itself an area where the measuring vehicle is supposed to operate is specified. It is not allowed to carry out a measurement under unfavorable weather conditions like raining, snowing or fog. In the first step of the measurement all four measuring photocells must be calibrated. The calibration procedure is as follows. The head of the calibrating photometer is placed very close to the measuring photocell. Both photo sensors measures illuminance and its values are recorded into a notebook. This procedure is executed on each single measuring photocell. After data processing an unique calibration coefficient $k_{i}$ will be assigned to each measuring photocell.

(1)

$$
k_{i}=\frac{E_{i k}}{E_{i}}
$$

where: $k_{i}$ - calibration coefficient of i-th photocell, $E_{i k}-$ illuminance measured by calibration photometer close to ith photocell, $E_{i}$ - illuminance measured by i-th photocell, I acquires values from 1 to 4 .

The measurement itself consists of following sub events. Driver leads the measuring vehicle at the beginning of the not yet measured logic segment using navigation in external screen. Then he goes thru this segment. The vehicle speed remains constantly $30 \mathrm{~km} / \mathrm{h}$. Measuring vehicle keeps moving (if possible) on a strait trajectory on the right side of the traffic lane. Operator inserts into a SW in notebook the beginning of the logic segment when he comes thru it. Since this moment SW automatically measures each 2 meters values of illuminance from all four photocells, GPS position and the values of temperature and humidity. SW starts the measurement each 2 meters thanks to the wheel rotation data provided by OBD II unit. Depending on the wheel rotation a time between each two measurement is counted so the distance between each two measuring points remains constant. Two meters of measuring distance responds to a $1 / 10$ of the lighting field length with $20 \mathrm{~m}$ of pole distance so it makes 10 measuring point per lighting field (according to the standard). Lighting fields with pole distance longer than $20 \mathrm{~m}$ have even more measuring points which make the measurement more accurate.

After setting a beginning of the new logic segment operator is asked to start inserting above-mentioned parameters. Lighting system geometry parameters and illuminated area parameters are according to the operator's professional estimation quantified. Other parameters have preset choice based on standard [3]. Together with these parameters a type of luminaire for each logic segment is also specified (if it is known). If operator hasn't got the information about the luminaire type in current logic segment a reference luminaire is set. Reference luminaire has the very similar light distribution curve to a luminaire commonly used in such road type. It is also possible to use reference luminaire when there are several different luminaires in a single logic segment without any obvious reason. Operator sets the end of the logic segment when one or more of observed parameters changes its value or when there is an end of the street. In the first case the end of one logic segment also means the beginning of another.

\section{Measured data evaluation}

When the whole measurement is over, the data from notebook are transferred into a central computer and further processed. In case one or more parameter weren't set during a measuring or it is necessary to check it, it is possible to use an in-car camera record. The appropriate parameter could be modified this way. Illuminance values from each of the four photocells are multiplied by its unique calibration coefficient $k_{i}$.

(2)

$$
E_{i j k a l}=k_{i} E_{i j}
$$

where: $E_{i j k a l}$ - calibrated illuminance of i-th photocell in the jth measuring point, $k_{i}$ - calibration coefficient of $\mathrm{i}$-th photocell, $E_{i j}$ - measured illuminance of i-th photocell in a jth measuring point.

For each logic segment following sub results are processed:

- average illuminance of the segment (not corrected)

- correction function system

- average illuminance (corrected)

- lighting class

- comparison between real and requested illuminance value

(3)

$$
E_{m}=\frac{\sum_{j=1}^{n} E_{1 j k a l}+E_{2 j k a l}+E_{3 j k a l}+E_{4 j k a l}}{4 n}
$$

where: $E_{m}$ - average segment illuminance, $E_{1 j k a l}, E_{2 j k a l}, E_{3 j k a l}$, $E_{4 j}$ kal - calibrated illuminance in j-th point of the measuring, $n$ - number of measured points in a logic segment.

From the lighting system geometry, illuminated area geometry and from the appropriate light distribution curve by using a special zonal flux method a correction functions system is created. Light distribution curves are processed by special SW application developed by experts from CTU, Department of Electrical Power Engineering. As an input this application uses ies files. Dependence of partial 
luminous flux on an angle in a plane perpendicular to the car movement direction makes the applications output.

Correction functions system is used to recalculate average calibrated illuminance measured in the height of $1,6 \mathrm{~m}$ above the road surface in the right part of the traffic lane into average (calibrated) illuminance measured at the road surface over the entire width of the traffic lane $E_{m} k o r$ (see Fig. 2). From the parameters of road surroundings and from traffic parameters of the logic segment a light class is defined according to [3]. According to the standard [4] those lighting classes are quantified by appropriate illuminance value.

Values of average corrected illuminance and required average illuminance are afterwards absolutely and relatively compared with each other. Partial result is the information about a quality of measured logic segment's lighting system.

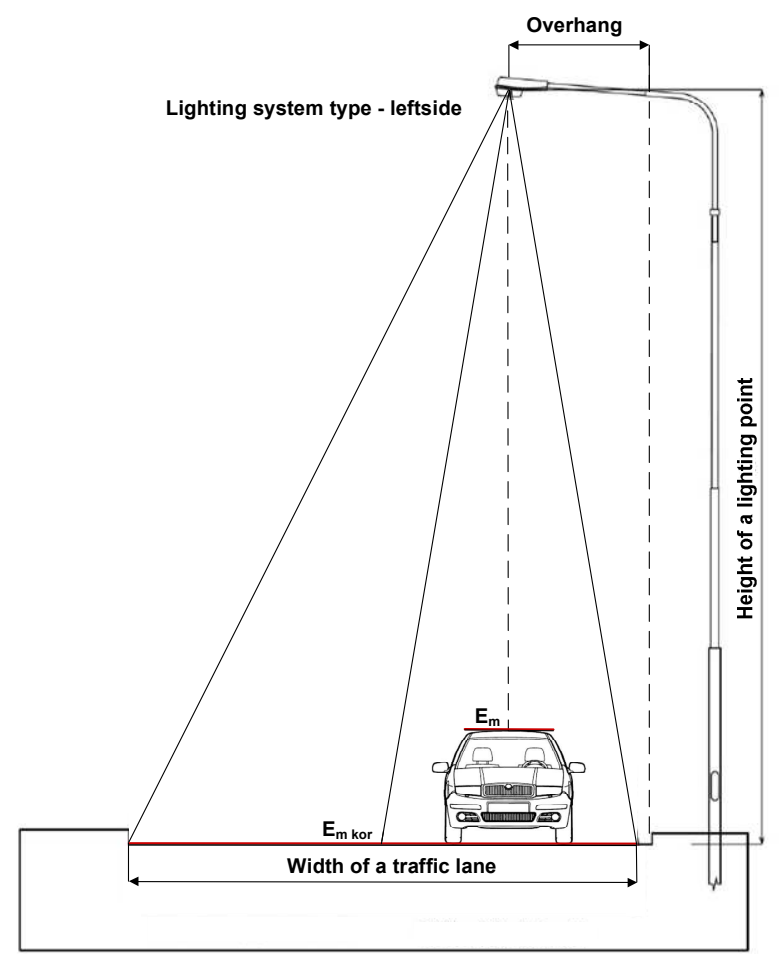

Fig. 2. Measured illuminance according to height and width of the street

All operations with measured data are in the database system executed automatically. There are two types of table outputs. Firstly it is a table for each single logic segment. Each row of the table corresponds to one logic segment. Besides the data set by operator it contains also:

- ID of logic segment

- GPS position and the time when the beginning and the end of the logic segment was set

- Calibration coefficient of each measuring photo cell

- Corrected and not corrected values of illuminance

- Required values of illuminance and the lighting classes

- Relative and absolute deviations between corrected and required illuminance

In the second type of the table each row represents one measuring point (illuminance of all four photo cells, GPS position, time of measurement, temperature and humidity).

\section{Visualization of results}

Final output of this project is a graphical visualization of chosen values from the logic segments table. Primary intent is to show a relative difference between corrected and required illuminance. However it is only a matter of processor's choice what other parameter will be visualized (for instance lighting class, type of luminaire, pole height, etc.). Visualized values are color distinguished according to chosen scale. Each logic segment is in SW ArcGIS connected with available street network using GPS positions of each measuring point. This modification gives to the measured data the shape of a measured street. Finally, the measured data are displayed onto an orthophoto map of chosen area. Without the possible connection with a street network measured data would be displayed only according to GPS position. Lack of accuracy of GPS would cause in that case difference between displayed results and orthophoto map. In case there is no street network available, special graphical functions are applied on the measured data.

\section{Measurement accuracy}

As already stated it wasn't the objective of this project to develop a measuring device with the highest accuracy class so the measurement could be comparable with the standard requirements. Total measurement uncertainty depends mostly on following factors:

- Uncertainty of each measuring element (photocell, photometer, etc.)

- Uncertainty when entering parameters of geometry, illuminated area and surroundings

- Other lighting sources contribution

- Climatic conditions (temperature, humidity)

- Spectrum of measured lighting sources and spectral responsibility of measuring elements (including calibration photometer)

- Difference between ideal and real light distribution curve of the luminaire (because of it's age or dirt)

- Difference between the light distribution curve of a real and a reference luminaire (if used)

- The accuracy of a measuring vehicle moving trajectory

- Non-homogeneity of the lighting system according to one logic segment

Thus it is very difficult to determine the whole uncertainty. According to the comparison of project's outputs and manual measuring it is possible to estimate the average project uncertainty as $\pm 15 \%$.

\section{LITERATURE}

[1] ČSN EN 13201-3 Road lighting - Part 3: Calculation of performance, May 2005

[2] ČSN EN 13201-4 Road lighting - Part 4: Methods of measuring lighting performance, May 2005

[3] ČSN CEN/TR 13201-1 Road lighting - Part 1: Selection of lighting classes, March 2007

[4] ČSN EN 13201-2 Road lighting - Part 2: Performance requirements, May 2005

Author: Ing. Jan Novotný, ELTODO, a.s., Novodvorská 1010/14, 142 01, Prague 4, Czech Republic, e-mail: novotnyj@eltodo.cz 